\title{
Pathologie des fondations superficielles sur sols argileux retour d'experience en Midi-Pyrenées
}

\section{JACQUARD \\ FONDASOL \\ BP 767}

84035 Avignon CEDEX 3 catherine.jacquard@rondasol.fi
Indépendamment des phénomènes de déformation des sols argileux liés autx variations de contraintes dues aux constructions d'ouvrages, le retrait etou le gonflement de certains sols argileux sont directement liés aux variations de leur teneur en eau.

L'amplitude de ces variations sera fonction de la nature de l'argile, de sa proportion dans le squelette des sols, de la structure de ce sol, de son épaisseur et bien entendu des gradients de succion ou d'hydratation qui luỉ seront imposés.

A partir d'une importante base de données sur les sols argileux de Midi-Pyrénées, ce document fait la synthèse des conmaissances de ces problèmes.

Mots-cles : retrait-gonflement- argiles gonflantespathologie des fondations superficielles- Région MidiPyrénées.

\section{Shallow foundations on clayey soils pathology out of experience in Midi-Pyrenees area}

\author{
廿 If Independenty of movements due to building and associated \\ pressures on soil most of clayey soils are susceptible to \\ shrinkage or swelling, which depend of moisture content \\ variations. \\ The oscillation of these variations depends on the type of clay \\ minerals, of its proportion contained in the soil, of the structure \\ of the soil, of the thickness of its layer, and of courne of the \\ gradients of suction or moisture imposed to it. \\ From a huge series of data on clayey solis in Midi-Pyrenees area, \\ this article is a synthesis of knowledge.
}

Key words: Shrinkage-swelling-5welling clays- shaliow foundations pathology-Midi-Pyrenees area

Ce document concerne spécifiquement la pathologie des fondations sur les formations molassiques on région Midj-Pyrénées, par retrait et gonflement. 
Cet article présente les moyens de caractériser le potentiel de retrait et de gonflement des sols argileux, établi à partir des données d'études de diagnostic géotechnique sur des maisons individuelles, effectuées par la société SORES entre 1992 et 1996 en région MidiPyrénées et dont les résultats nous ont été communiqués par André Bedin, alors directeur de la SORES, que je tiens ici à remercier tout particulièrement.

\section{1}

\section{Reconnaître les sols gonflants}

\section{1}

\section{Étude minéralogique}

La connaissance de Ja minêralogie des argiles constitutives d'un sol peut se faire à partir du diffractomètre à rayon X et du microscope à balayage électronique (MEB). Ĺacquisition de ces matériels est coûteuse, et les bureaux d'étude géotechnique n'en sont généralement pas équipés. Ces identifications permettent néanmoins de caractériser la nature, la structure, et l'état de porosité de l'argile, et donc d'avoir une idée du potentiel de retrait et de gonflement du sol, mais sans aucune indication sur l'amplitude et la quantification du phénomène.

\section{2}

\section{Analyses granulométrique et sédimentométrique}

Ces mesures de la répartition pondérale de la dimension des grałns du sol sont nécessaires pour quantifier la proportion d'argile: \% passant al tamis de $2 \mu m$ (également appelé $\mathrm{C}_{2}$ ), obtenue par la sédimentométrie.

\section{Limites d'Atterberg}

Ces mesures donnent la limite de plasticité $W_{\text {p }}$ la limite de liquidité $W_{4}$, et l'indice de plasticité $I_{\mathrm{P}}=W_{\mathrm{I}}-\mathrm{W}_{\mathrm{P}}$

Toutes les recherches effectuées montrent que les sols gonflants se trouvent classés au-dessus de la droite A dans le diagramme de Gasagrande, ce qui apporte peu d'information sur' le risque potentiel d'une argile : on indique sur la figure 1 les limites d'Alterberg en regard des coefficients de gonflement Cg (voir \$ 1.5) mesurés en région Midi-PyTénées. Les sols peuvent présenter un fort risque de gonflement dès lors que $W_{L}>35$ pour les points au-dessus de la droite de Casagrande. Sur les sols courants, D. Lautrin (1987) montre que, dès lors que le sol présente moins de 25 à $30 \%$ d'argile, les limites d'Atterberg ne sont pas significatives de la composition minéralogique des argiles du sol, ni de la sensibilité à l'eau de ce sol.

\section{4}

\section{Surface spécifique et valeur au bleu de méthylène VBS}

La surface spécifique est la surface cumulée des plaquettes dans une certaine quantité de sol ; elle s'exprime en $\mathrm{m}^{2} / \mathrm{g}$. La surface spécifique externe correspond au niveau interparticulaire, tandis que la surface spécifique interne correspond au niveau interfoliaire. Mlite

70 à $140 \mathrm{~m}^{2} / \mathrm{g}$ interparticulaire (filler calcaire $\sim 1 \mathrm{~m}^{2} / \mathrm{g}$ )

Montmorillonite $800 \mathrm{~m}^{2} / \mathrm{g}$ interfoliaire+ interparticulaire

L'essai le plus courant, et le plus simple pour mesu rer cette surface est actuellement l'essai d'absomption au bleu de méthylène qui représente le mieux la surface active des argiles :

$\mathrm{Sa}=20,93$ VBS $(20,93=$ surface correspondant à $1 \mathrm{~cm}^{3}$ de bleu de méthylène).

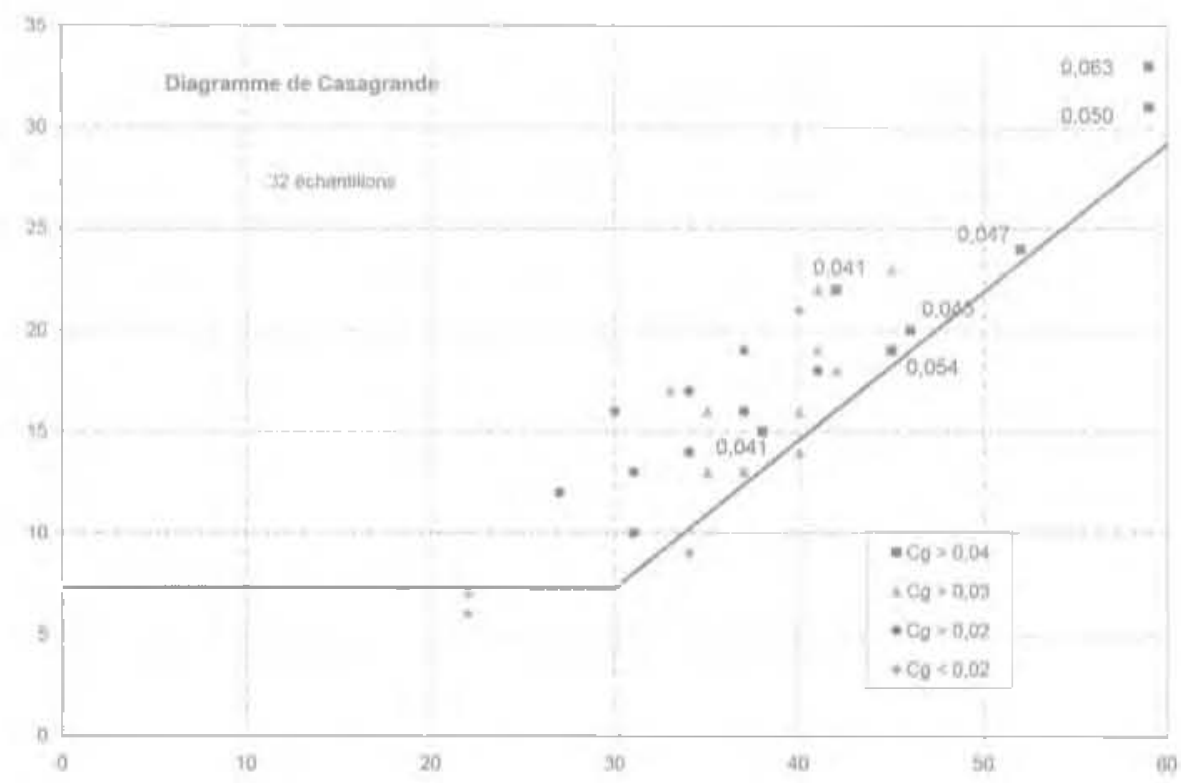

FG. 1 Limites d'Atterberg et coefficient de gonflernent $\mathrm{Cg}$ pour les sols d'origine molassique, région MidjPyrénées. 
Cette mesure donne ainsi la Guantité et l'activité de la fraction argileuse. Les recherches et études de D. Lautrin (1989) l'ont conduit à une classification à par"tir du pourcentage de passant à $2 \mu \mathrm{m}(\mathrm{C}$ ) et la valeur au bleu (VBS) sur le matériau 0/D (voir tableau I) :

$$
A_{\mathrm{CB}}=100 \mathrm{VBS} / \mathrm{C}_{2}
$$

tableaul Activité des argiles (d'après D. Lautrin).

\begin{tabular}{c|c}
\hline $\mathbf{A}_{C H}$ & Activitè des argiles \\
\hline$>18$ & Nocives \\
\hline 13 à 18 & Très actives \\
\hline 8 à 13 & Actives \\
\hline 5 à 8 & Normales \\
\hline 3à 5 & Peu actives \\
\hline 1 à 3 & Inactives \\
\hline$<1$ & Sols non argileux \\
\hline
\end{tabular}

Cette classification permet également d'avoir une indication sur la nature de l'argile constitutive du sol.

On rappelle qu'il y a lieu de distinguer la valeur au bleu VB mesurée sur la fraction fine du sol passant à $80 \mu \mathrm{m}$ ), at la valeur de bleu VBS mesurée par les géotechniciens (NFP 94-068) correspondant à la valeur de bleu de la fraction $0 / 50 \mathrm{~mm}$ du sol. Ces deux mesures sont identiques pour des sols purement limoneux et argileux (sols fins).

\section{5}

\section{Mesure de la pression et du coefficient de gonflement $\mathrm{Cg}$}

La pression de gonflement est la mesure de la pression exercée par le matériau mis en présence d'eau, lorsque tout déplacement est empêché. Cette pression dépend de l'état hydrique du sol au moment de l'essai. Il existe d'autres modes opératoires que nous ne développerons pas ici. En laboratoire, on réalise l'essai à l'oedomètre sur échantillon intact, par mesure de la variation du volume des vides en fonction du logarithme de la contrainte appliquée.

On sait, depuis G. Philipponnat (1978), relativement bien corréler le risque pathologique, さे la pente de retour lors du déchargement Cg (voir tableau II).

TRLLA II Risque de gonflement (d'après G. Philipponnat).

\begin{tabular}{c|c}
\hline $\begin{array}{c}\text { Coefficient de } \\
\text { gonflement Cg }\end{array}$ & fisque de gonflement \\
\hline$>0,05$ & Certain \\
\hline $0,05-0,04$ & Très grand \\
\hline $0,03-0,04$ & Grand \\
\hline $0,02-0,03$ & Possible \\
\hline$<0,02$ & Peu probable \\
\hline
\end{tabular}

L'essai oedométrique révèle également la surconsolidation liée à l'évaporation et à l’évapotranspiration, par le rapport de la contrainte de préconsolidation $\sigma^{\prime}$ à la contrainte à la profondeư de prélèvement $\sigma_{\text {wa }}^{\prime}$ (voir figure 2),

La pression de gonflement doit être comparée à la contrainte de service au sol sous fondation (et dallage) : les sols de la région toulousaine ont des pressions de gonflement de l'ordre de 100 à $150 \mathrm{kPa}$ pour une dessiccation correspondant à un degré de saturation de 85 à $90 \%$.

Il a été établí pour des argiles d'altération molassique une bonne corrélation entre ce coefficient de gonflement $\mathrm{Cg}$ et la valeur de bleu (voir figure 3), ainsi qu'entre $\mathrm{Cg}$ et le produit VBS $\times \mathrm{C}_{2}$ (voir figure 4, établie par Bedin,1999 sur le graphicue de classification de Magnan, 1989). On pourra ainsi retenir que, sans dispositif de protection adapté, dès lors que VBS $\times C_{2}>100$, le risque de désordre est certain et, lorsque VBS $\times \mathrm{C}_{2}<50$, le risque est peu probable.

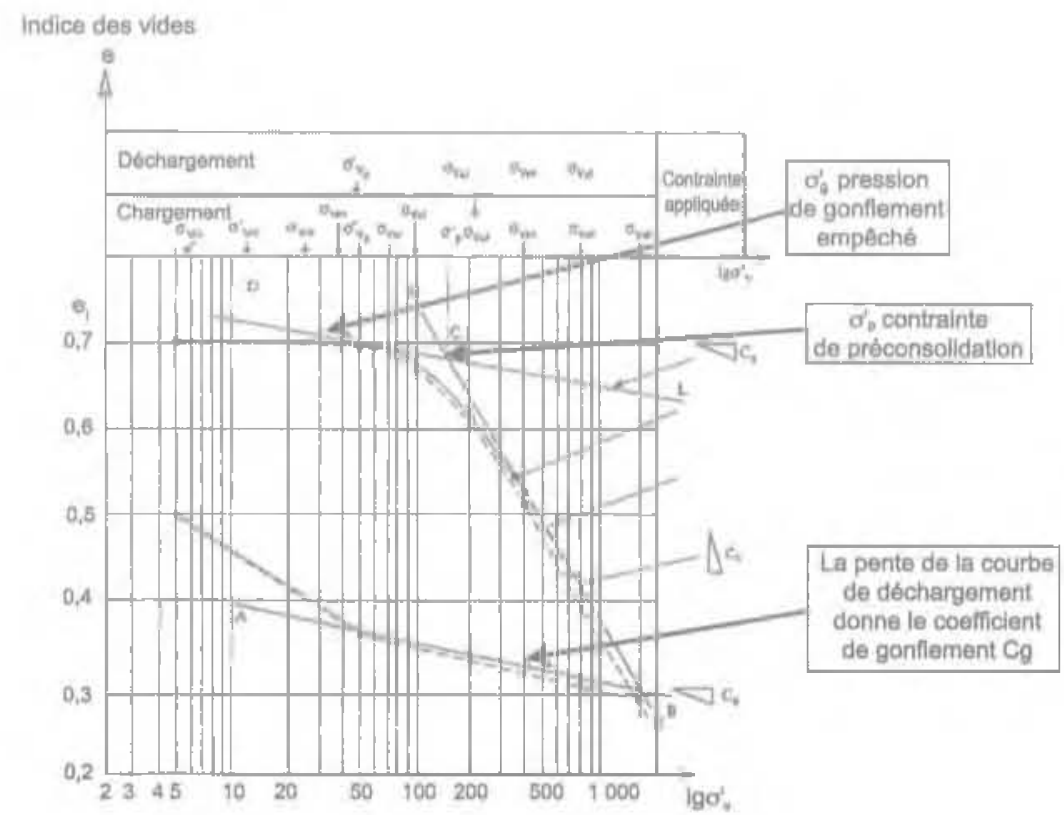




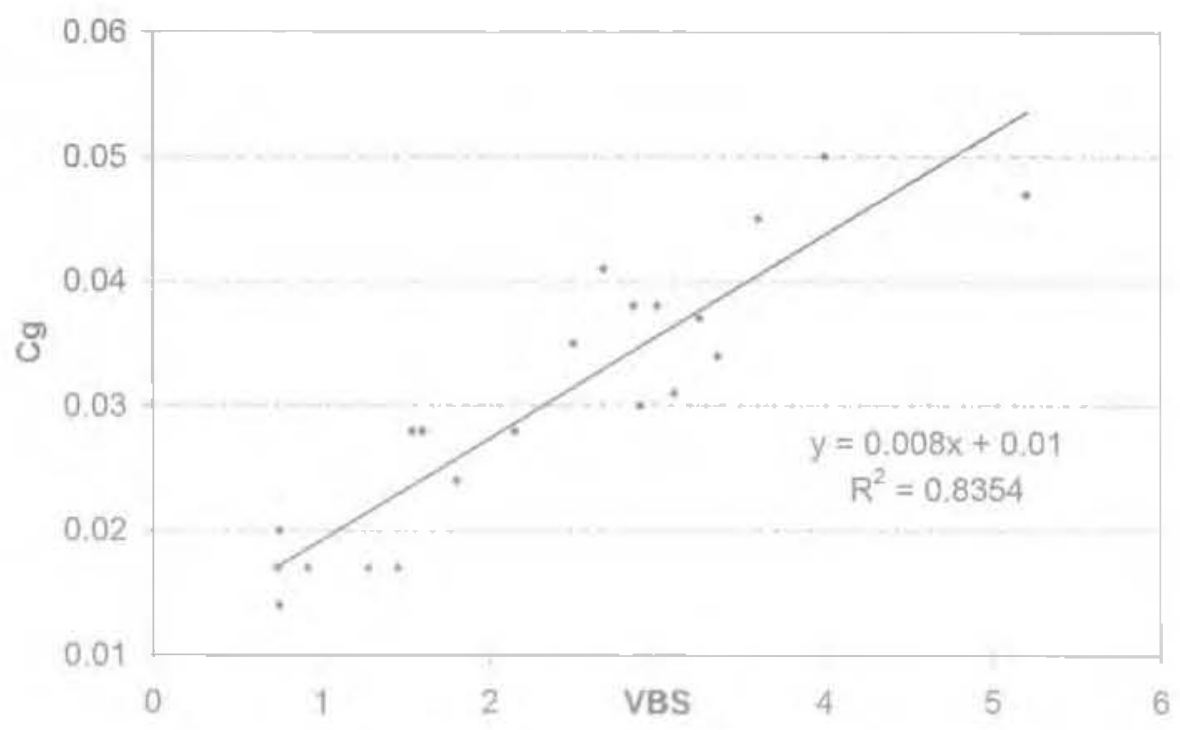

FkG. 3 Sols de lâ région Midi-Pyrénées ; cortélation VRS/Cg.

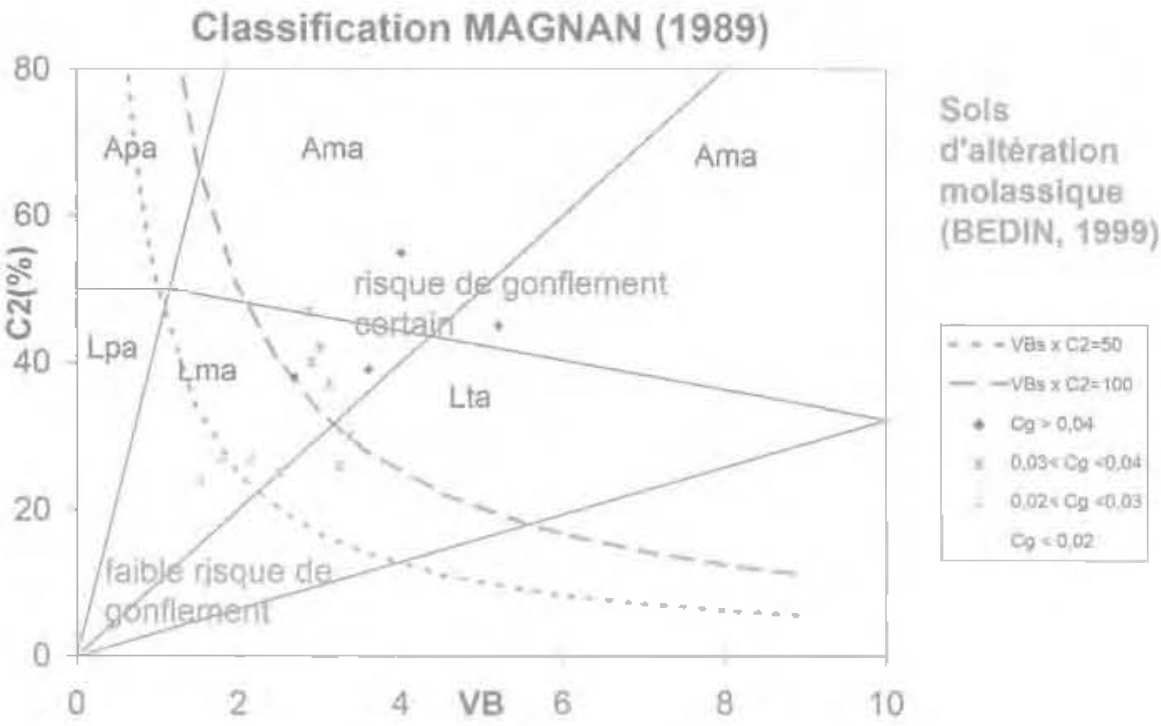

ตc 4 Sols de la région Midi-Pyrénées : classification Magnan $\left(\mathrm{VB}-\mathrm{C}_{2}\right.$ ) et corrélation Bedin (VBs $\times \mathrm{C}_{2}$ )

\section{6}

\section{Mesure du retrait}

Cet essaî, réalisé sur un échantillon intact (XP P94060-2), permet de déterminer la limite de retrait effective du sol ( $\mathrm{W}$ ), c'est à dire la limite en deçà de laquelle la perte d'eau n'entraîne plus de rétractance, et où la matrice argileuse du sol se désature. La pente de la droite permet aussi de quantifier les variations dimensionnelles entre l'état de teneur en eau naturelle $W_{\text {nat }}$ et un état de dessiccation donné, cette pente s'appelle le retrait linéaire : Rl (voir figure 5).

Le seuil de risque est avéré lorsque $\mathrm{Rl}>0,3$ associée à une large plage de retrait potentiel ( $W_{n a t}-W_{r 0}>10$ ).

Cet essai permet d"estimer le tassement des fondations, dû à látractance par dessiccation.

Sur les sols d'altération d’origine molassique, les corrélations du retrait linéaire avec la valeur de bleu (VBS) ou l'activité de I'argile $\left(A_{C B}\right.$ ) ne sont pas signifi- catives. En revanche, on a pu indiquer le potentiel de retrait, en fonction du poids volumique sec et de l'êtat de teneur en eau (voir ci-après).

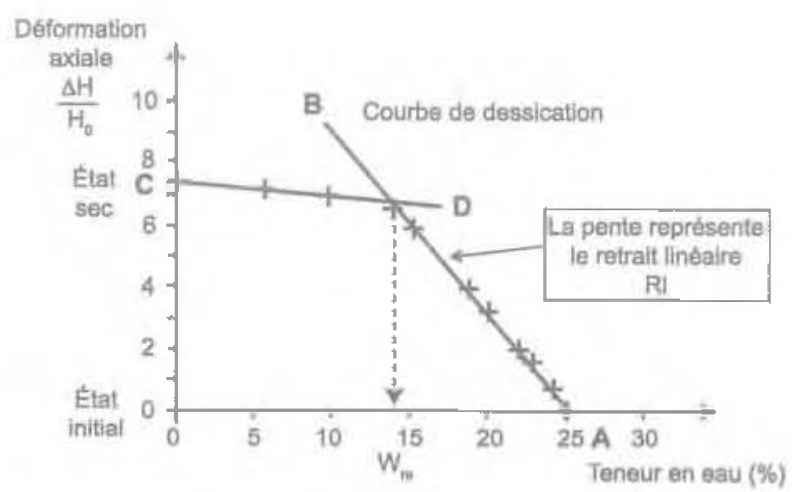

na. I Procès-verbal d'essai de retrait. 


\section{Mesure de la masse volumique du sol et de sa teneur en eau}

C'est un essa de laboratoire des plus simples, mais qui nécessite le prélèvement d'un échantillon intact ; it donne :

- le poids volumique du sol sec $\gamma_{\text {d }}$ et la teneur en eau du sol W;

- l'état de dessiccation des sols par le calcul du degré de saturation Sr déduit de ces deux mesures. Sa valeur normale à partir de 50 à $70 \mathrm{~cm}$ de profondeur est de 92 à $98 \%$, et naturellement $100 \%$ à proximité et sous la nappe. Des valeurs inférieures à $80 \%$ reflètent une dessiccation importante.

Sur les sols d'origine molassique étudiés, le diagramme $W-\gamma_{d}$, permet de caractériser la zone des sols à risque (voir figure 6].

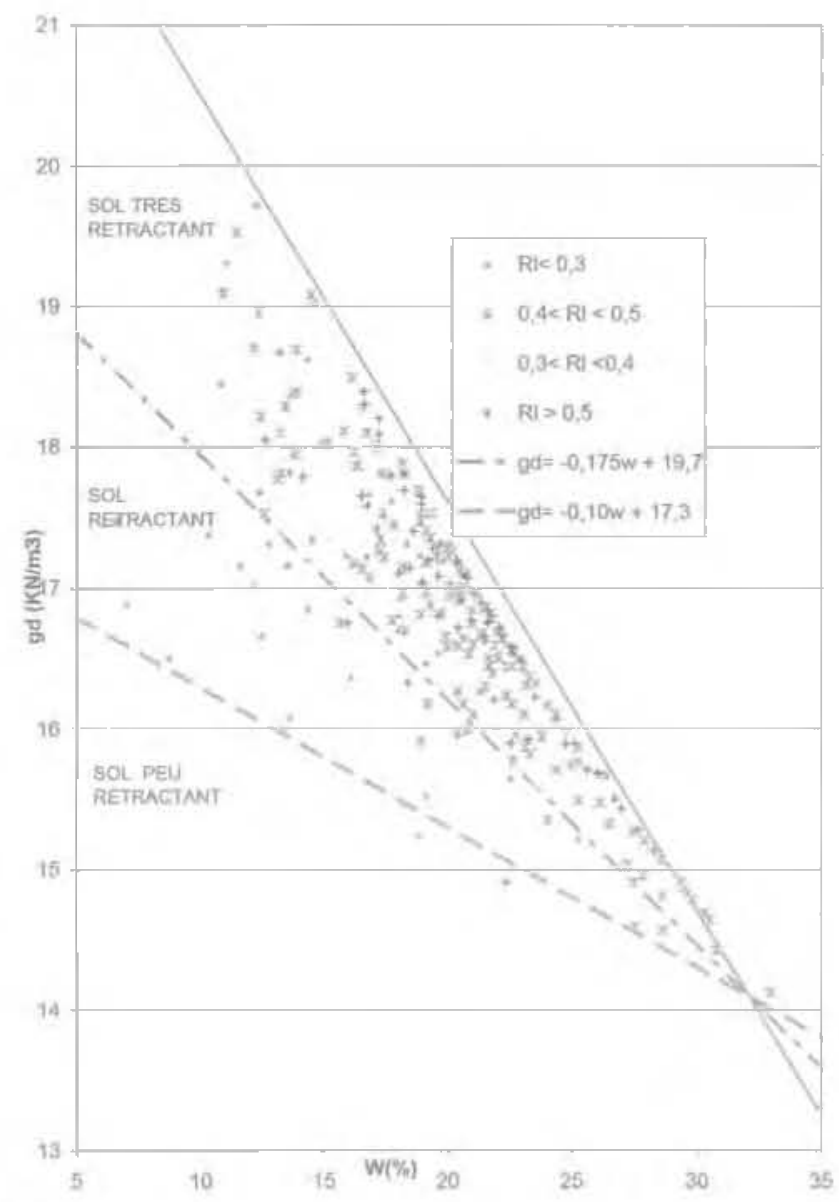

WF. Diagramme $w-\gamma_{\text {d }}$ pour les sols d'origine molassique, région Midi-Pyrénées.

Les analyses statistiques effectuées en région toulousaine ont par ailleurs permis de tenter une approche de la sensibilité à partir d'un coefficient de dessiccation Cd caractérisant la variation de poids volumicue sec pour une variation de teneur en eau imposée à un échantillon intact (Bedin, 1999) :

$$
\mathrm{C}_{\mathrm{d}}=\Delta \gamma_{d} / \Delta \mathrm{W}
$$

On peut ainsi indiquer en fonction de la valeur : $\mathrm{C}_{\mathrm{d}}>0,185$ : sols très sensibles;

$\mathrm{C}_{\mathrm{d}}<0,12$; sols peu sensibles.
Cet essał nécessite plusieurs mesures de poids volumique en laissant baisser la teneur en eau ; mode opératoire qui se rapproche de celui de l'essal de dessiccation.

\section{8}

\section{Essais in situ}

En aucune manière les essais in situ ne permettent de qualifier ou quantifier ces phénomènes d'argiles actives, tout au pliss peuvent-ils renseigner sur l'homogénéité de dessiccation autour d'une construction et la profondeur d'action de cette dessiccation.

Deux appareils sont couramment utilisés : le pénétromètre et le pressiomètre.

Les essais de pénétration dynamique sont des essais simples et peu onéreux pour connaître la profondeur de dessiccation (Fìg. 7a et 7b), mais ce sont des essais aveugles vis-è-vis de la rature des sols traversés, et significatifs jusqu'à une profondeur limitée, du fait des frottements parasites sur le train de tiges. Le pénétromètre statique donne des résultats équivalents, mais les camions lourds généralement utilisés les rendent peu pratiques pour caractériser la pathologie des maisons individuelles. Ces essais doivent être réalisés en parallểe à des sondages permettant de connaître la nature des sols ainsi traversés.

Les essais pressiométriques donnent la pression limite Pl du sol, et son module pressiométrique $\mathrm{E}_{\mathrm{M}}$. La pression limite est peu affectée par la dessiccation, en revanche celle-ci va faire augmenter le module : lorsque le rapport $\mathrm{E}_{\mathrm{M}} / \mathrm{Pl}$ est, dans des argiles, supérjeur à 14 ou 16, il y a surconsolidation du sol liée à la dessiccation. C'est par ailleurs le seul essai qui permette de déterminer le frottement latéral gs à prendre en compte pour le dimensionnement des micropieux, lorsqu'une reprise en sous-ceuvre s'avère nécessaire.

\section{?}

\section{Profil hydrique d'un sol et perturba- tions créées par les constructions}

Sous une construction 11 y aura perturbation du profil hydrique du centre vers le bord. Au centre de la construction, le degré de saturation aura tendance à croître, et la nappe à remonter si elle est proche. En l'absence de nappe, l'eau aura tendance à migrer de la périphérie vers le centre après chaque période pluvieuse, et au centre les sols se satureront progressivement.

En périphérie, les sols vont subir les variations climatiques, les angles étant les plus exposés.

C'est ainsi que, sur un sol gonflant, on obtient à moyen terme (5 \&े 7 ans) :

- un maxim»m de gonflement au centre (mouvement lent et continu) :

- un gonflement minimum dans les angles ;

- un gonflement intemédiaire le long des murs.

Au départ la situation peut être inverse en fonction de l'époque de construction. En particulier si la construction est faite en saison sèche, c'est la périphérie qui aura tendance à gonfler au départ, alors que le centre ne bougera pas. 


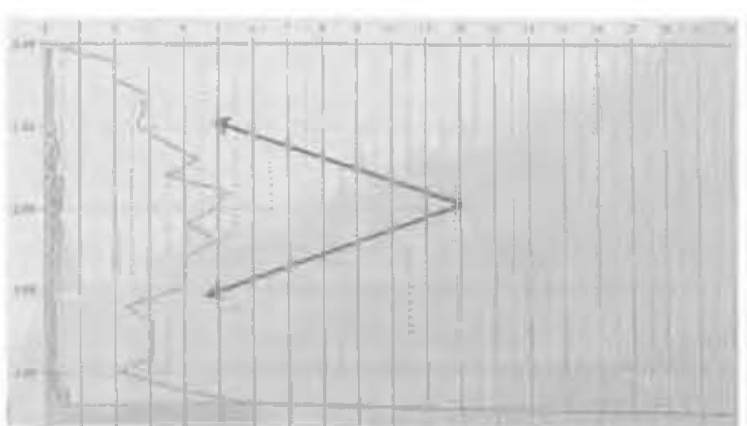

(a)

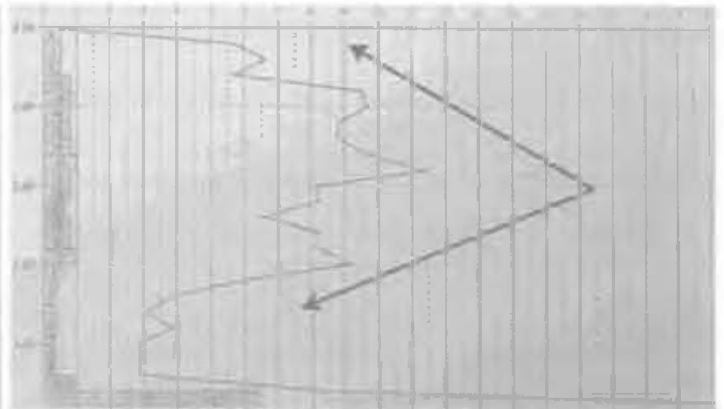

(b)
Les constructions sut sol argileux sensibles à l'eau seront donc soumises à des mouvements différentiels à court terme et à long terme. La forme des fissures dépendra de la rigidité de la structure et pourra évoluer avec le temps.

\section{3}

\section{Désordres liés aux sols sensibles}

\section{1}

\section{Directions principales des fissures}

Les deux formes principales de fissuration sont des déformations de flexion et de cisalilement.

Les fissures de cisaillement vont se rencontrer sur les structures type portique, les fissures de flexion sur les murs insufisamment rigides au sommet ou à la base (fissure en queue de billard).

En général, s’il s'agit de sols gonflants, le mourement est alternatif avec une évolution progressive générale. Ces mouvements sont très néfastes pour les constructions; elles produisent à long terme une extension du réseau des fissures, contribuant au démantèlement de la structure dont la rigidité et la capacité de résistance ala tassement différentiel điminue au cours du temps.

La dessiccation se traduit souvent par une ouverture au niveau de l'arase sanitaire.

Il n'est pas possible pour le spécialiste de confondre les désordres dus aux phénomènes de retrait/gonflement avec ceux provenant de tassements de consoliciation ou de mouvements de grande ampleur comme les glissements ou les fluages.

Cependant, certaines fissures de structure (retrait thermique) peuvent se confondre avec des fissures de fondation; de même sur les carrelages il est souvent difficile de visu, de distinguer les fissures inhérentes aux mouvements des fondations de celles propres à un défaut de pose (effet d'étau ou déformation de l'isolant).

Contrairement aux tassements de consolidation, les mouvements provenant de sols gonflants ne se stabili-

\section{2}

\section{Valeurs admissibles des déformations différentielles}

Les constructions modernes élancées et rigides s'accorderont mal aux tassements différentiels et la valeur maximale du 1/1000 de la portée devrât être respectée pour la pérennité des structures

En ce qui concerne une maison individuelle classique, avec un ou pas de plancher, des chaînages verticaux d'angle et un chaînage horizontal sous charpente, sa rigidité est quasi nulle vis-ầ-vis des tassements différentiels. Les dénivellations doivent être limitées à 1/500 de la portée, avec un seułl maximum de $1 \mathrm{~cm}$ pour éviter les désordres sur les cloisonnements.

Sur sol gonflant, cette valeur est extrêmement diffficile $\mathrm{a}$ respecter en raison des variations d'humidité sous la construction et des variations cycliques sur les bords, et des faibles contraintes apportées au sol.

\section{3}

\section{Déformation des dallages}

Il s'agit d'une fondation superficielle peu chargée et qui par conséquent souffrina le plus des problèmes, d'autant qu'il se cumule le problème d'exécution (nature de la forme. compactage sur les bords, ferraillage, épaisseur du béton, et problème des isolants).

La pathologie sera d'autant plus importante que le daliage est dissocié des murs; dans certains cas, seul l'intérieur de la construction est sinistré, alors que la coque extêriteure est intacte.

\section{4}

\section{Étude statistique de la pathologie en Midi-Pyrénées}

Cette étude statistique a été menée entre 1997 et 1999 par SORES sur environ 400 cas pathologiques en région Midi-Pyrénées. 


\section{Paramètres concernant la construction}

\section{4 .9}

\section{Aģe de la construction}

Les sinistres concernent les constuctions relativement récentes (10 à 30 ans) mais environ $16 \%$ concernent les constructions anclenres (> 100 ans) dont les murs se prolongent en fondation sans élargissement (voir figure 8).

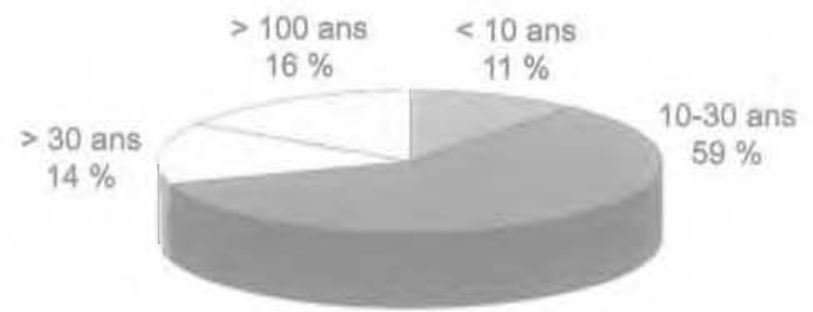

Re. 8 Répartition de l'âge des constructions affectées.

\section{Type de constructions (nombre de niveaux)}

Pratiquement, seuls les bâtiments $\mathrm{R}+0$ et $\mathrm{R}+1$ sont affectés (Fig. 9), ceci pour deux raisons :

- la transmission des charges au sol est faible et la dissipation des contraintes est très rapide en profondeur;

- la rigidité des bâtiments à plusieurs niveaux est généralement plus grande, avec possibilité de report de charge en cas de tassement différentìel.

\section{Thin}

\section{La rigidité de la structure}

Aucune construction traditionnelle de maison individuelle n'est dimensionnée avec possibilité de dénivellations d'appuis, tels que tassements différentiels. En revanche, une construction en $\mathrm{R}+0$ comportant deux planchers aura moins ã souffrir et développera des fissures (fissures horizontales) beaucoup moins pénalisantes qu"une maison sur dallage dissocié des murs, et plafond suspendu.

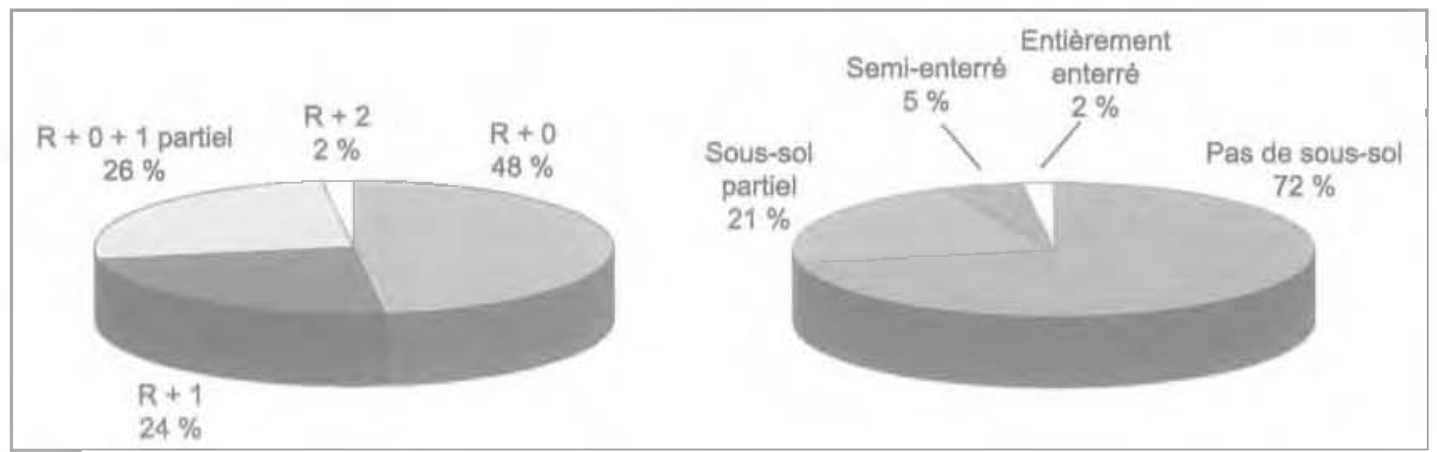

Types de bátiments concernés par la pathologie.

\section{Les sous-sols}

Les désordres affectent principalement les bâtiments sans sous-sols et ceux avec un sous-sol partiel, qu“il s'agisse de terTain horizontal ou en pente, le sozs-sol atteint des niveaux hors perturbation hydrique, ce qui n'est pas le cas pour les autres fondations.

Les désordres très classiques se retrouvent dans le cas de sous-sol partiel au raccordement de la partie en sous-sol avec une partie en rez-de-chaussée, dans la zone traversant la foulle du sous-sol.

Dans la hauteur du sous-sol la pression de gonflement peut s'additionner à la poussée des terres, et aussi créer des efforts dissymétriques sur les voiles enterrés.

\section{1 .5}

\section{Encastrement des fondations}

Sur l'étude statistique menée dans la région MidiPyrénées (Fig. 10), il apparaît que la pathologie conceme pour plus de $50 \%$ des cas des fondations comprises entre 0,5 et $0,9 \mathrm{~m}$ : une profondeur d'encastrement de $1 \mathrm{~m}$ ne paraît donc pas suffisante pour rester à l'abri des variations hydriques.

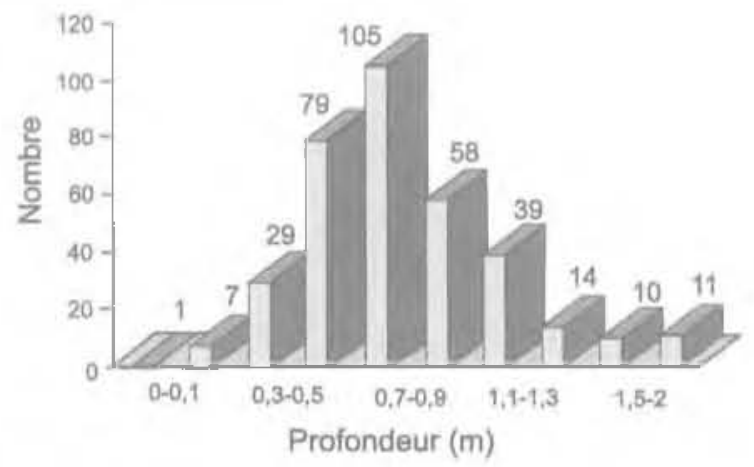

Fa. 10 Profondeur des fondations des maisons sinistrées.

Dans environ $10 \%$ des cas, la profondeur hors-gel n'est pas respectée : il s'agit essentiellement de maisons 
anciennes pour lesquelles le mur se prolonge de quelques dizaines de centimètres dans le sol.

Dans $10 \%$ des cas - encastrement inférieur à $50 \mathrm{~cm}$-, la malfaçon est le paramètre déterminant : la sécheresse vient révéler le problème.

Actuellement on constate un approfondissement graduel de la dessiccation quí sur un même site est passée de $0,80 \mathrm{~m}$ après la sécheresse de 1989-1991, à $1,3 \mathrm{~m}$ après la canicule de 2003 .

L'encastrement minimal, dit de fond de cave (ou sous le niveau du vide sanitaire), sur des sols argileux ne devrait donc pas être inférieur à $1,30 \mathrm{~m}$, avec de plus une bonne protection périphérique (trottoir et chenaux), et sur un terrain correctement assaini.

En présence d'arbres, cet encastrement doit étre beaucoup plus important.

L'excentrement de la maçonnerie sur la fondation peut être le paramètre déterminant du sinistre, associé à un mur haut nor contreventé.

Par ailleurs, et même s'il n'y a pas de règles formelles, on considère qu'une Fondation béton de $\mathrm{H}<60 \mathrm{~cm}$ et/ou $B<50 \mathrm{~cm}$ ne peut jouer son rôle de répartition des efforts, en fonction de l'hétérogénéité de portance du sol d’assise. Si les risques existent, le ferraillage doit être supérieur au minimum prescrit par les règles du béton armé.

\section{1 .6}

\section{Période de construction}

Il est constaté que si la construction est faite en période très humide ou très sèche, les déformations des angles les plus exposés peuvent se produire rapidement (année suivante).

A une période intermédiaire, l'évolution est très lente, elle entrâne dans la structure des contraintes parasites qui se développent peu à peu jusqúà la limite de rupture.

\section{9}

\section{Paramètres statistiques concernant le sol}

\section{Identification des sols}

A partir des essais classiques d'identification des sols à savoir granulométrie et sédimentométrie donnant le pourcentage d'argile $\mathrm{C}_{2}$, et la valeur ata bleu de méthylè̀ne VBS, il a été mis en évidence les corrélations suivantes sur les argiles du Midi-Pyrénées:

- suivant la classification proposée par Magnan, les sols étudiés sont essentiellement des limons argileux ; l'identification (VBS, $\mathrm{C}_{2}$ ) donne une bonne indication sur la sensibilité des sols au phénomène de gonflement (Fig. 4), mais ne permet de quantifier le risque : pour le faire il faut réaliser des essais spécifiques : mesure de la pression et du coefficient de gonflement à l'oedomètre par exemple;

- il n'a pu être mis en évidence une corrélation nette entre VBS, IP, $\mathrm{C}_{2}$ et retrait linéaire; en revanche on peut indiquer la sensibilité à la dessiccation à partir de

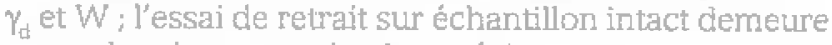
cependant la mesure lą plus précise.

\section{8.}

\section{Risque de gonflement}

Sur l'ensemble des cas étudiés, la répartition des coefficients de gonflement est une gaussienne centrée sur : $\mathrm{Cg}=$ 0,03-0,04 (Fig. 11). Majoritairement, la pression de gonflement $\mathrm{Pg}$ est faible [Fig. 11) et souvent même, inférieure à la contrainte de service, ce qui implique dans ces cas, la non réversibilité du phénomène de retrait, et conditionne la méthode de réparation.

\section{9 .9}

\section{Retralt linéaire}

Sur l'ensemble des cas étudiés, la répartition du retrait linéaire indique plus de $86 \%$ de valeurs supérieures à 0,3 (voir figure 12), ce qui confirme le risque avéré au-delà de ce seuilı mais doit être tempéré par la plage de variation hydrique potentiel.
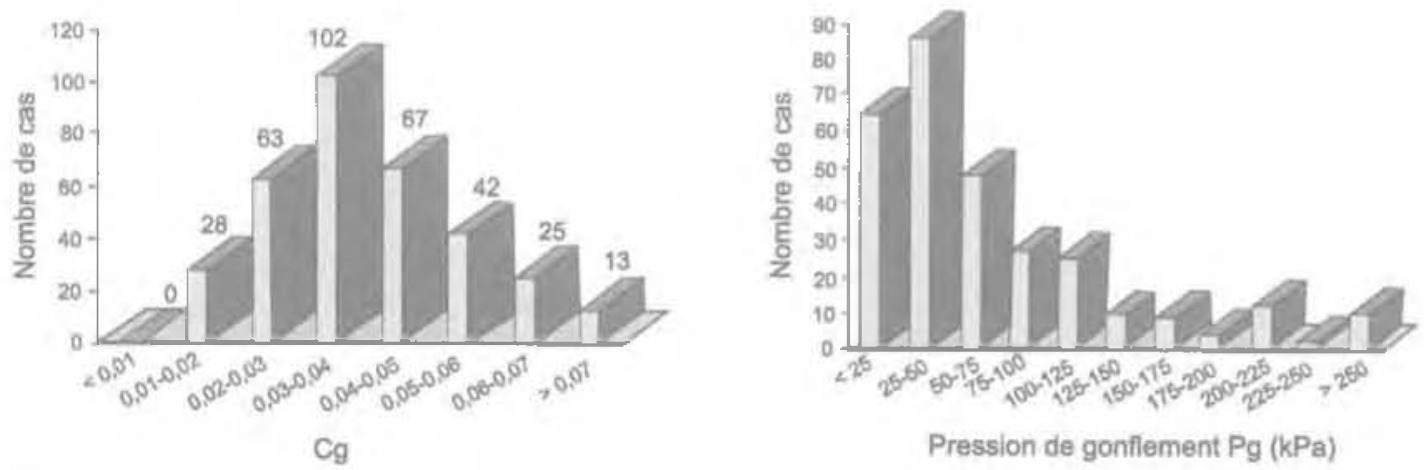

FG. 11 Sols de la région Midi-Pyrénées : coefiicients de gonflement Cg et pressions de gonflement Pg mesurés. 


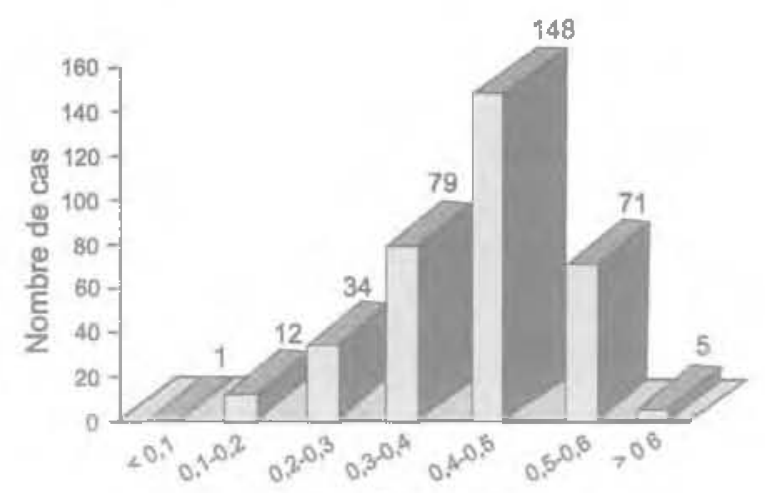

Retrait linéaire RI

Fa 19 Sols de la région Midi-Pyrénées: retrait linéaire mesuré.

\section{9}

\section{Lithologie et dessiccation}

Plus les sols argileux de couverture sont épais, plus les risques liés ã la dessiccation sont importants, surtout en présence de végétation. Si les essais de pénétration détectent bien les zones surconsolidées subissant la dessiccation, ils doivent être combinés avec un sondage permettant l'établissement d'un profil lithologique (tarière ou carottage), voire d'un profil hydrique.

\section{2 .5}

\section{Portance des sols, compressibilité}

La contrainte de semice sous les fondations des maisons individuelles est toujours faible, puisqu'en général une contrainte admissible de 100 à $120 \mathrm{kPa}$ est suffisante. L'indice de compression Cc mesuré à l'oedomètre est dans une majorité des cas compris entre 0,1 et 0,2 ce qui correspond à des sols faiblement à moyennement compressibles, de plus généralement dans un état plutôt surconsolidé. Il n'y a donc pas de problème de compressibilité sur ce type de sols.

\section{8 .6}

\section{Homogénéité de l'assise des fondations}

Il est rare d'avoir d'importantes variations lithologiques sous une construction sur terrain plat et horizontal ; en revanche, sur terrain en pente, le modelage des terres va créer l'hétérogénéité : les fondations aval doivent être approfondies pour le respect de l'homogénéité des fondations. De même, avec un sous-sol partiel, les terrassements peuvent découvrir des sols de nature hétérogène.

\section{Pente du terrain}

Le cycle retrait/gonflement participe sur la pente au phénomène de reptation, celui-ci est considérablement accéléré en période d'accidents climatiques par approfondissement de la fissuration de retrait.

\section{3}

\section{Les actions extérieures}

\subsection{1}

\section{Les variations hydriques}

Elles peuvent être provoquées par :

- l'assainissement général en périohérie vìs à vis des eaux météoriques et des eaux de nappe (topographie générale du site) :

- la protection des Fondations (trottoir-chenaux) ;

- l'urbanisation quj peut provoquer dans certains cas, le gonflement des argiles surconsolidées si la nappe est proche; dans d'autres, au contraire, un retrait par dessiccation des eaux evacuées au pluvial, ou un rabattement génêral de la nappe.

On peut également rencontrer des actions accidentelles provoquant des variations de teneur en eau, génératrices de désordres : fuites de conduites, pertes d'eau des fosses, chauffage.

\section{La végétation}

La végétation va considérablement approfondir le profil hydrique du terrain vierge et donc amplifier le phénomène de retrait.

Par ailleurs, l'action de succion des ractnes est sajsonnière. De plus, les racines se dirigent dans les zones humides, c'est-à-dire sous la construction : leur action est ainsi géométriquement limitée sous la construction et va contribuer au tassement différentiel.

Les arbres sont classés par leur coefficient d'agressivité, le plus agressif étant le chêne également en Midi-Pyrénées (voir tableau III), avec des racines ou radicelles susceptibles de descendre parfois à $10 \mathrm{~m}$ de profondeur! En fail, tout arbre situé à moins de 1 à 1,5 fols sa hauteur adulte est agressif vis-ả-vis des fondations superficielles sur sols argileux. Les haies mệme de faible hauteur sont également très nocives.

Sur les cas étudiés en Midi Pyrénées, la répartition des espèces végétales ayant provoqué ou aggravé le

zangell II Espèces d'arbres agressives (sulvant Cutler, Thee roots and buildings, 1989).

\begin{tabular}{|c|c|c|c|}
\hline Espèce & $\begin{array}{c}\text { Hauteur } \\
\text { moyenne (m) }\end{array}$ & $\begin{array}{c}\text { Distance des } \\
\text { désordres }\end{array}$ & $\begin{array}{c}\text { Coefficient } \\
\text { dagressivite }\end{array}$ \\
\hline Chêne & 20 & 30 & 5.5 \\
\hline Peuplier et frêne & $18 \mathrm{a} 25$ & 20 à 30 & 2,7 \\
\hline Acacia, marronnier, tilleut, saule & 20 & 20 & 1.4 \\
\hline Aubépine, hêtre, platane & 10 à 25 & 15 & $\$, 0$ \\
\hline Pommier, poirier & 10 & 10 & 0,8 \\
\hline Cerisier, prunier, bouleau, cyprès, orne & 10 à 20 & 10 \& 20 & 0,4 \\
\hline
\end{tabular}


sinistre, présenté figure 13 correspond aux espèces habituellement agressives.

Les fondations neuves, tout comme les reprises en sous-ceuve devront descendre en dessous du système racinaire, et prendre en compte les effets parasites générés par leur action cyclique. On peut également limiter leur effet par interposition d"écrans anti-racines, qui doivent descendre également sous la base du système racinaire, ce qui rend parfois difficile et oné reuse leur mise en celve.

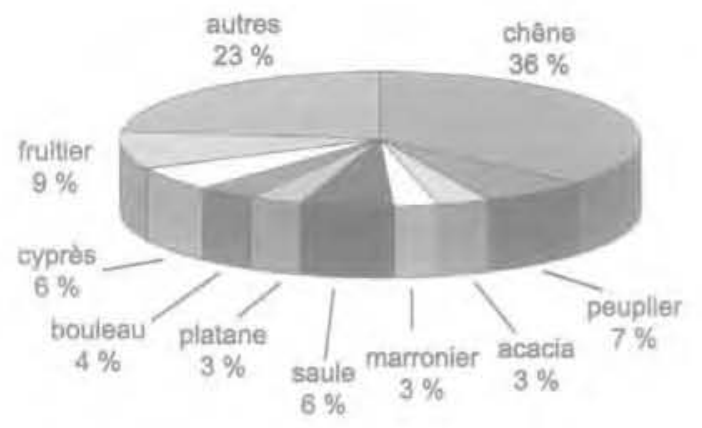

nG. 13 Répartition des espèces végétales, causes de pathologie en Midi-Pyrênées.

Bien souvent la proximité des arbres est l'élément déterminant des désordres, la sécheresse n'étant plus qu'un paramètre aggravant; ce phénomène est caractéristique lorsque les mouvements sont limités aux façades exposées aux arbres, alors que la sécheresse généralise les désordres sur l'ensemble de la construction.

\section{4}

\section{Récapitulatif des causes déteminantes}

Les causes déterminantes retenues pour expliquer la pathologie sont rếsumées dans le tableau IV. Globalement sur 389 cas pathologiques étudiés, la sensibilité des sols d'assise au phénomène de retrait par dessiccation est un des paramètres déterminants dans $92 \%$ des cas, mais l'influence d'une végétation proche des constructions est un phénomène aggravant considérable ( $70 \%$ des cas). Dans $50 \%$ des cas, on observe que les fondations ont une profondeur d'encastrement de moins de $90 \mathrm{~cm}$, dont $27 \%$ sont réanmoins supérieures à $70 \mathrm{~cm}$, c'est-à-dire hors gel, ce quil indique clajrement qu'en dehors d'une protection efficace contre les variations hydriques une profondeur d'assise plus importante est nécessaire pour éviter des désordres.

taBLFAUN Causes d'apparition de désordres idonnées, région toulousaine).

\begin{tabular}{l|c}
\hline \multicolumn{1}{c|}{ Całıses déterminantes du désordre } & Nombre de cas \\
\hline Rétractance des sols par dessiccation & 358 \\
\hline Végétation néfaste & 271 \\
\hline Protection insuffisente des fondations & 196 \\
\hline Pente du terrain & 165 \\
\hline Manque de rigidité des structures & 138 \\
\hline Fondation trop légère & 101 \\
\hline Présence d'un sous-sol partiel & 79 \\
\hline Construction par étapes & 58 \\
\hline Vétusté & 55 \\
\hline
\end{tabular}

On constate qu'il y a rarement une cause unique à l'origine d'une pathologie; la sécheresse est souvent le phénomène déclenchant, mais fréquemment amplifié par des causes secondaires concomitantes : dispositions constructives non adaptées sur un terrain en pente ( $42 \%$ des désordres), manque de rigidité des structures (35\%).

\section{Bibliographie}

Bigot G., Zehrouni M.I. - Retrait-gonflement et tassement des sols fins. Bulletin de hiaison des laboratoires des ponts et chaussées $n^{\circ} 229$, novembre-décembre 2000.

Lautrin D. - Une procédure rapide đ̛identification des argiles. Bulletin de liaison des laboratoires des ponts ct chaussées $n^{0} 152$, novembre-décembre 1987.
Lautrin D. - Utilisation pratique des paramètres dérivés de l'essai au bleu de méthylène dans les problèmes de génie civil. Bulletin de liaison des laboratoires des ponts et chaussêes n ${ }^{\circ} 160$, février mars 1989.

Magnan a.-P. Youssefian G - Essai au bleu de méthylène et classification géotechnfque des sols- Bulletin de laison des labo- ratoires des ponts et chaussées $\eta^{\circ} 159$ janvier-février 1989

Philipponnat G. - Désordres dus à la présence de sols gonflants dans la Région parisienne. Annales de I'TBTP n ${ }^{\mathrm{B}} 364$, septembre 1978 .

Philipponnat G. - Retrait-gonflement des argiles, proposition de méthodologieRevise française de géotechnique $n^{\circ} 57$. 1991. 